Article

\title{
Endocrine Disruption: Structural Interactions of Androgen Receptor against Di(2-ethylhexyl) Phthalate and Its Metabolites
}

\author{
Mohd Amin Beg ${ }^{1, *}$ (D) and Ishfaq Ahmad Sheikh ${ }^{1,2}$ \\ 1 King Fahd Medical Research Center, King Abdulaziz University, Jeddah 21589, Saudi Arabia; \\ iasheikh@kau.edu.sa \\ 2 Department of Medical Laboratory Technology, Faculty of Applied Medical Sciences, \\ King Abdulaziz University, Jeddah 21589, Saudi Arabia \\ * Correspondence: mbeg@kau.edu.sa or mabeg51@gmail.com; Tel.: +966-552822451
}

Received: 8 November 2020; Accepted: 6 December 2020; Published: 8 December 2020

\begin{abstract}
Diethylhexyl phthalate (DEHP) is a commonly used plasticizer in the manufacture of polyvinyl chloride plastics for household and commercial use. DEHP is a ubiquitous ecocontaminant and causes developmental and reproductive problems in children and adults. After exposure, DEHP is metabolized by endogenous hydrolysis and oxidation into the primary metabolite, mono-(2-ethylhexyl) phthalate (MEHP), and the secondary metabolites, mono-(2-ethyl-5-hydroxhexyl)phthalate (5-OH-MEHP), mono-(2-ethyl-5-oxohexyl) phthalate (5-oxo-MEHP), mono-(2-ethyl-5-carboxypentyl) phthalate (5-cx-MEPP), and mono-[(2-carboxymethyl)hexyl] phthalate (2-cx-MMHP). Very few studies have been reported on the adverse effects of DEHP metabolites, and the available information indicates that the metabolites might also be equally or more active as compared to the parent compound. In the present study, induced fit docking was used for structural binding characterization of the above five DEHP metabolites with androgen receptor (AR) to predict the potential endocrine-disrupting effects of these metabolites in AR signaling. All the DEHP metabolites interacted with the ligand-binding pocket of AR forming amino-acid residue interactions, hydrogen bonding, and pi-pi interactions. The binding energy of DEHP with AR was similar to that of native ligand testosterone. The amino-acid residue interactions of DEHP metabolites had $91-100 \%$ similarity compared to that of testosterone. In addition, all the DEHP metabolites and testosterone showed a common hydrogen bonding interaction with amino-acid Arg-752 of AR. Taken together, the structural binding data in the present study suggested the potential for DEHP metabolites to disrupt AR signaling, which may lead to androgen-related reproductive dysfunction.
\end{abstract}

Keywords: androgen receptor; DEHP; MEHP; 5-OH-MEHP; 5-oxo-MEHP; 5-cx-MEPP; 2-cx-MMHP; docking

\section{Introduction}

Di(2-ethylhexyl) phthalate (DEHP) is a synthetic chemical prepared commercially from phthalic anhydride with an excess of 2-ethylhexanol in the presence of an acid catalyst and is the single largest plasticizer by production volume used in the world [1-3]. Even though the production and use of DEHP are declining due to the reported adverse effects on human health, it is forecasted to constitute more than one-third of about 10 million tons of the total plasticizer market in the world in $2024[4,5]$. The major use of DEHP is for increasing the flexibility of polyvinyl chloride plastics in which it may constitute up to $40 \%$ of the weight [2]. The main (95\%) applications of DEHP are for polymer uses such as toys, footwear, shower curtains, wire and cable coating, electronic component parts, molds, 
wall covering, paper coating, roofing, flooring, industrial applications, medical devices, etc. In contrast, minor applications are in paints, inks, adhesives, dielectric fluids, etc. [6].

DEHP is a known endocrine-disrupting chemical (EDC) and is associated with reproductive, endocrine, and other disorders in humans such as infertility, endometriosis, thyroid hormone disruption, testicular dysgenesis syndrome, diabetes, obesity, cancer, behavioral issues, etc. [1,7,8]. DEHP has been officially prohibited for use in the manufacture of children's toys in the United States since 2009 and has been categorized as a Group 2B human carcinogen by the United States Environmental Protection Agency. In addition, the agency has recently designated DEHP as a high-priority substance for risk evaluation based on its assessment of the unreasonable risk of injury to health and environment, including susceptible subpopulations [9]. Moreover, due to the adverse effects, DEHP is considered a priority and/or hazardous pollutant in many other countries, including Canada and the Netherlands [10]. DEHP has been designated as a dangerous chemical by the European Union and is regarded as human fertility impairing and developmental toxic (category 2) chemical [11]. The Japan Society for Occupational Health has designated DEHP as a group 1 chemical on the basis of human reproductive toxicity [12].

DEHP is ubiquitously present in the environment, and major exposure from DEHP occurs through food contamination as it migrates from plastic containers and wrappings during processing and storage [2,13,14]. In one of the recent studies [15], a high majority (74\%) of 72 common food products such as pork, chicken, infant foods, and other food items in the United States were contaminated with detectable levels of DEHP. Exposure also occurs through medical devices during assisted respiration, cardiopulmonary bypass, kidney dialysis, blood transfusions, incubators, etc. [16]. In addition, people working in factories that manufacture or use DEHP are subjected to significant occupational aerosol exposure. The systemic personal exposure of DEHP is measured by analysis of DEHP or its breakdown products in the urine, blood, or other body fluids [17]. After gaining access, DEHP undergoes hydrolysis in the human body to form a primary metabolite, mono-(2-ethylhexyl)phthalate (MEHP) [18]. MEHP then undergoes hydroxylation and oxidation and forms several secondary metabolites, which are excreted from the body with urine, sweat, milk, semen, and saliva [19]. Four major DEHP secondary metabolites are mono(2-ethyl-5-hydroxyhexyl)phthalate (5-OH-MEHP) and mono(2-ethyl-5-oxyhexyl)phthalate (5-oxo-MEHP), mono(2-ethyl-5-carboxypentyl)phthalate (5-cx-MEPP) and mono [2-(carboxymethyl)hexyl]phthalate (2-cx-MMHP) $[18,20]$. The structural illustration of the major metabolites of DEHP is given in Figure 1. After a single dose of DEHP in adult human volunteers, 5-OH-MEHP was the major metabolite in urine until $12 \mathrm{~h}$ and 5-cx-MEPP was the major urinary metabolite from 12-24 h, whereas, the major metabolite after $24 \mathrm{~h}$ was 2-cx-MMHP [20]. About $70 \%$ of the dose was excreted in urine after $24 \mathrm{~h}$ constituting 5-OH-MEHP (23.3\%), 5-cx-MEPP (18.5\%), 5-oxo-MEHP (15.0\%), MEHP (5.9\%), and 2-cx-MMHP (4.2\%). In contrast, biomonitoring of newborns in neonatal intensive care has revealed that 5-cx-MEPP accounted for the majority $(60-83 \%)$ and 5-OH-MEHP for 5-15\% of the urinary DEHP metabolites [21,22].

Analyses of human body fluids such as urine [13], cord blood [23], amniotic fluid [24], follicular fluid [25], and milk and blood [26] have revealed detectable levels of DEHP metabolites. The potential adverse effects of DEHP are, in fact, thought to be more due to its metabolites; secondary metabolites are about 100 fold more embryo-toxic than MEHP $[27,28]$. In several other studies, reproductive and developmental problems such as preterm birth, altered reproductive hormone levels, hypospadias, cryptorchidism, anogenital distance anomalies and reduced gestational age for male babies, intellectual and motor development in children, adulthood infertility problems in men such as lower semen volume and sperm concentrations were associated with in utero exposure with DEHP and higher maternal serum levels of MEHP, 5-OH-MEHP, 5-oxo-MEHP, and 5-cx-MEPP [19,29]. In addition, higher production of reactive oxygen species, higher sperm apoptosis, and lower sperm concentration and motility in polyvinyl chloride workers was associated with higher MEHP, 5-OH-MEHP, and 5-oxo-MEHP levels in urine $[19,30]$. 


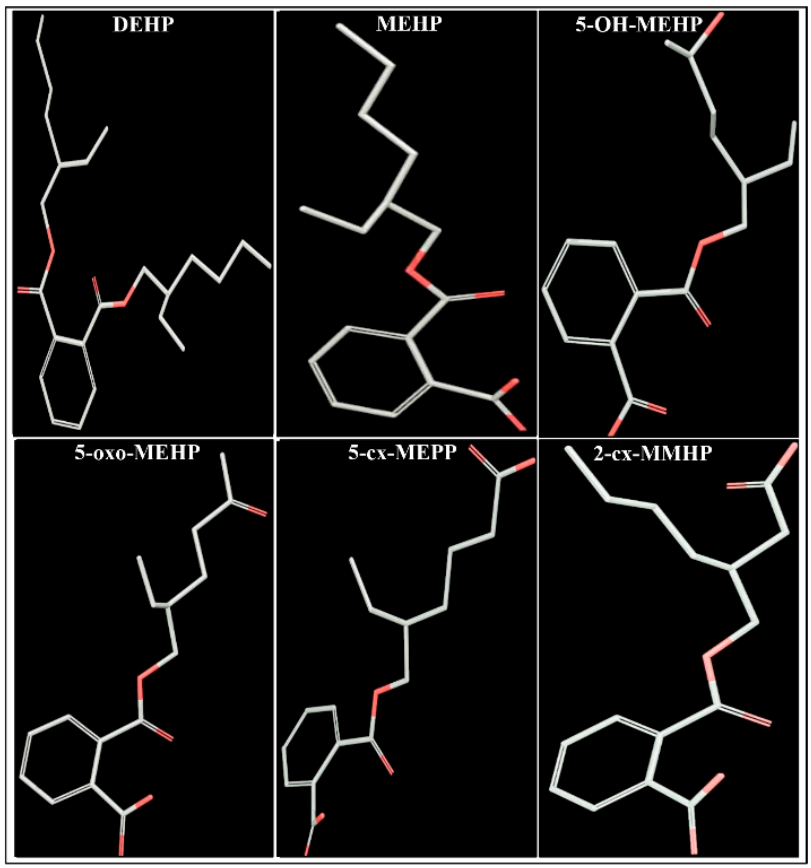

Figure 1. Three-dimensional representation of di-(2-ethylhexyl) phthalate (DEHP) and its five major metabolites, mono-(2-ethylhexyl)phthalate (MEHP), mono-(2-ethyl-5-hydroxyhexyl) phthalate (5-OH-MEHP), mono-(2-ethyl-5-oxohexyl)phthalate (5-oxo-MEHP), mono-(2-ethyl-5-carboxypentyl) phthalate (5-cx-MEPP), and mono-[2-(carboxymethyl)hexyl]phthalate (2-cx-MMHP).

In hospitalized intensive care patients, the DEHP exposure could reach very high levels. For example, the urinary levels of 5-OH-MEHP and 5-oxo-MEHP in babies under neonatal intensive care were reported in the range of $1-5 \mu \mathrm{g} / \mathrm{mL}[21,31]$. These exposures may continue for days or even weeks, thus could have severe adverse effects on the health of children and adults. The EDCs such as DEHP are thought to cause adverse effects in the body through interactions with steroid hormone receptors, hormone transport proteins, and steroid hormone enzymatic pathways [32]. Very few studies have been reported on the potential adverse effects of endogenous DEHP metabolites on human body functions. Available in vitro and structural studies on selective DEHP metabolites with androgen receptor (AR) have reported conflicting results [33-36]. Widespread environmental distribution, commercial use, and human exposure of DEHP warrant a thorough toxicological investigation of its endogenous metabolites. The current structure-based study on the interactions of DEHP metabolites with AR was done to help in understanding the potential disrupting activity of DEHP metabolites in androgen signaling. The study used molecular docking simulation to delineate the binding interactions of the five major endogenous metabolites of DEHP, i.e., MEHP, 5-OH-MEHP, 5-oxo-MEHP, 5-cx-MEPP, and 2-cx-MMHP with AR and differential comparisons with natural AR ligand testosterone for the binding pattern and the interacting residues.

\section{Materials and Methods}

The 2-dimensional structures of 6 ligands, i.e., DEHP, MEHP, 5-OH-MEHP, 5-oxo-MEHP, 5-cx-MEPP, and 2-cx-MMHP were drawn using Maestro 10.3 (Maestro, version 10.3, Schrodinger, LLC, New York, NY, USA, 2015) and this was followed by conversion of 2-dimensional structures into 3-dimensional structures (Figure 1). The structure was also searched in the PubChem compound database (https://pubchem.ncbi.nlm.nih.gov/) for identification and confirmation. The PubChem compound identities and abbreviations are presented in Table 1. For docking experiments of these ligands with AR, Schrodinger 2015 suite with Maestro 10.3 (graphical user interface) software (Schrodinger, LLC, New York, NY, USA, 2015) was used [37]. 
Table 1. Ligand name, common abbreviations, PubChem IDs and Chemical Abstract Service Registration Number (CAS No.) of di(2-ethylhexyl) phthalate and its five major endogenous metabolites.

\begin{tabular}{ccccc}
\hline Serial No. & Name & Abbreviation & PubChem ID & CAS No \\
\hline 1 & Di(2-ethylhexyl)phthalate & DEHP & 8343 & $117-81-7$ \\
2 & Mono-(2-ethylhexyl)phthalate & MEHP & 20393 & $4376-20-9$ \\
3 & Mono-(2-ethyl-5-hydroxyhexyl)phthalate & 5-OH-MEHP & 170295 & $40321-99-1$ \\
4 & Mono-(2-ethyl-5-oxohexyl)phthalate & 5-oxo-MEHP & 119096 & $40321-98-0$ \\
5 & Mono-(2-ethyl-5-carboxypentyl)phthalate & 5-cx-MEPP & 148386 & $40809-41-4$ \\
6 & Mono-[2-(carboxymethyl)hexyl]phthalate & 2-cx-MMHP & 187353 & $82975-93-7$ \\
\hline
\end{tabular}

\subsection{Protein Preparation}

The database Protein Data Bank (PDB; http://www.rcsb.org/) was explored to obtain the crystal structure complex of human AR (PDB code: 2AM9) co-complexed with natural ligand testosterone (TST) at resolution of $1.64 \AA$. The crystal structure was prepared for docking analysis using the protein preparation wizard workflow of Schrodinger Glide (Schrodinger suite 2015-3; Schrodinger, LLC) as described earlier [37]. Briefly, the crystal structure of the AR complex was imported to the docking program Glide and prepared by removing the water molecules, adding the hydrogen atoms and charges, and building loops and missing side chains using the protein preparation wizard workflow, OPLS-2005 force field, and Prime 3.0 module software. Then, the optimization of hydrogen bonds was done, and finally, a geometry optimization was performed to a maximum root-mean-square deviation (rmsd) of $0.30 \AA$. The natural ligand TST in the crystal structure was selected for the generation of grid boxes and used for docking of the ligands.

\subsection{Ligand Preparation}

The 2-dimensional structures of DEHP metabolites were drawn using Maestro 10.3 (Maestro, version 10.3, Schrodinger, LLC), and this was followed by conversion of 2-dimensional structures into 3-dimensional structures (Figure 1), as described previously [37]. LigPrep module (Schrodinger 2015: LigPrep, version 3.1, Schrodinger, LLC) was used to prepare the DEHP ligands, and OPLS-2005 force field software was used for obtaining correct molecular geometries and ionization at biological pH 7.4.

\subsection{Induced Fit Docking}

Schrodinger's Induced Fit Docking (IFD) module was utilized for experiments on docking simulations of AR with DEHP and its 5 metabolites. The prediction of accurate conformational changes in protein upon ligand binding was an issue that needed to be addressed to simulate the ligand-binding more precisely. The IDF method addressed this problem to some extent as it induced flexibility in the protein ligand-binding pocket during the docking experiment. The methodology of IFD has been described previously [37]. Briefly, DEHP metabolites were incorporated as starting geometries into the IFD software, which had the calculating capability of sampling the minor changes in the backbone structure as well as robust conformational changes in the side chains [38]. Docking of the ligand occurred into an ensemble of the binding protein conformations during the performance of a softened-potential docking in the first IFD stage. Subsequently, complex minimization for the highest-ranked pose was performed where ligand, as well as the binding sites, were free to move.

\subsection{Binding Affinity Calculations}

The ligand-binding affinity calculations of above-indicated phthalate plasticizer (DEHP) and its major metabolites for AR were performed using the Prime module of Schrodinger 2015 with molecular mechanics generalized born-surface area (MMGB-SA) function as described [37]. The MMGB-SA function was one of the best methodologies to be pursued and was a middle ground between the fast but inaccurate docking simulations and the free-energy perturbation, which gave the best results but it was very costly from a computational time perspective. 


\section{Results}

DEHP and its five major endogenous metabolites, i.e., MEHP, 5-OH-MEHP, 5-oxo-MEHP, 5-cx-MEPP, and 2-cx-MMHP, were successfully subjected to docking simulation with AR. The successful accomplishment ensued into the stable placement of all the ligands in the AR ligand-binding pocket. Several interaction poses were generated for each ligand during the docking procedure in the binding domain of AR, and the most representative stable docking poses for each ligand based on the result of cumulative analysis of docking parameters, including amino acid interactions in the ligand-binding pocket, which were visualized using Maestro as graphical user interface and binding energy estimation calculated by the MMGB-SA function, which was considered for further analysis. The resulting docking poses, amino-acid interactions, and analyses are presented for each compound along with the native ligand, TST (Figures 2-7, Tables 2 and 3).

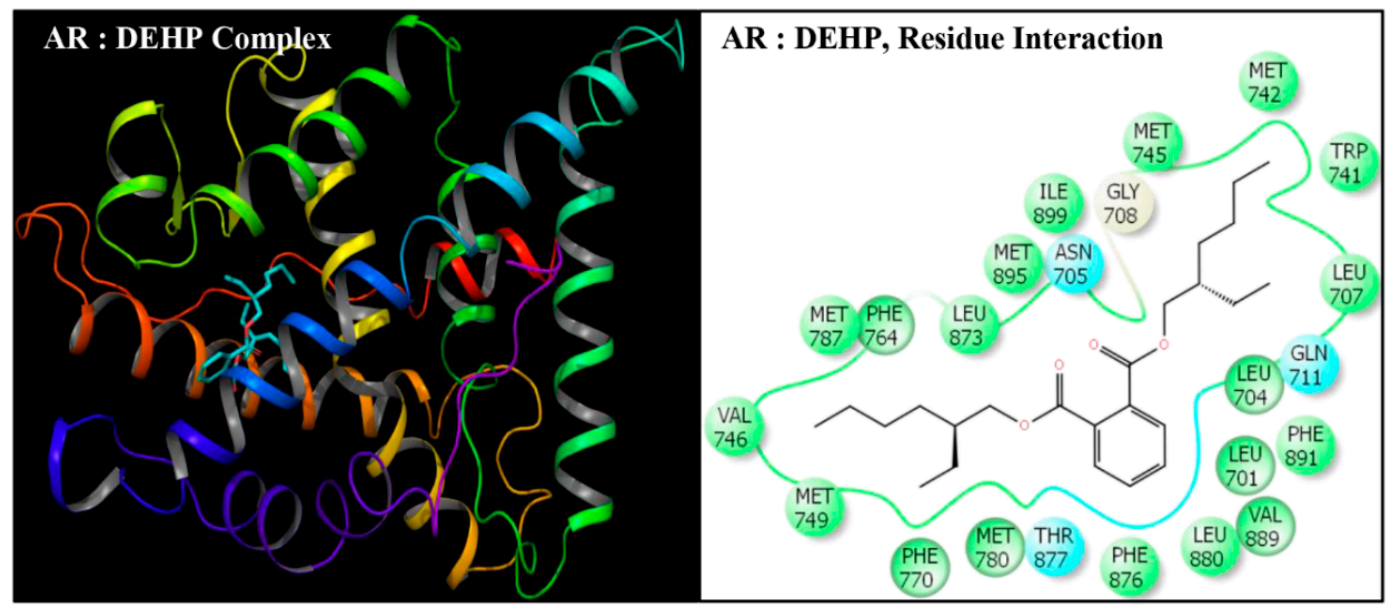

Figure 2. Ribbon diagram representing the docking pose of human androgen receptor (AR) with di(2-ethylhexyl) phthalate (DEHP) (left panel). The interactions of ligand (DEHP) with amino-acid residues in the binding pocket of AR (right panel).

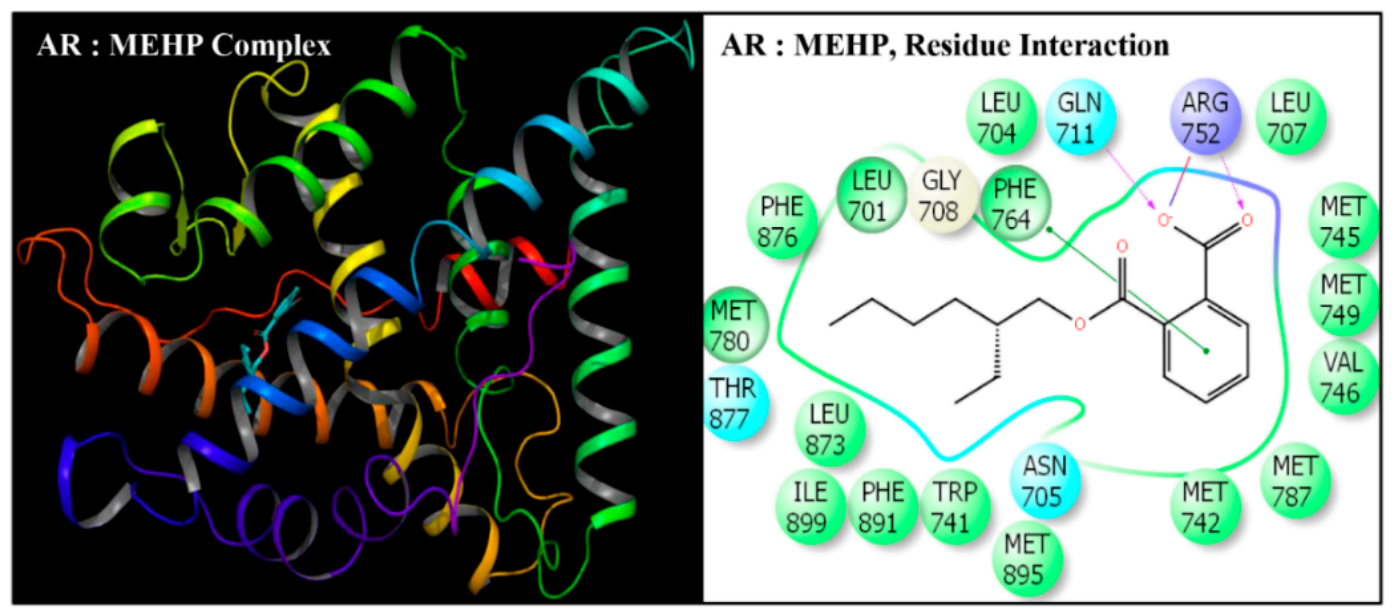

Figure 3. Ribbon diagram representing the docking pose of human androgen receptor (AR) with mono-(2-ethylhexyl)phthalate (MEHP) (left panel). The interactions of ligand (MEHP) with amino-acid residues in the binding pocket of AR (right panel). 


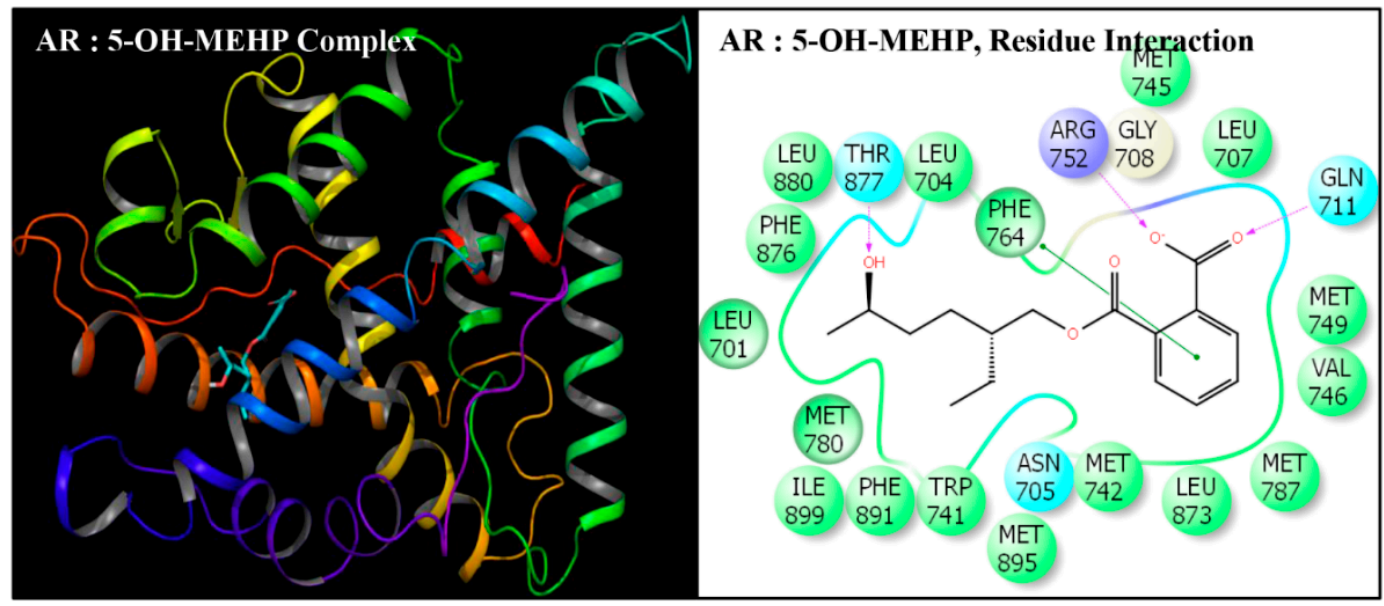

Figure 4. Ribbon diagram representing the docking pose of human androgen receptor (AR) with mono-(2-ethyl-5-hydroxyhexyl)phthalate (5-OH-MEHP) (left panel). The interactions of ligand (5-OH-MEHP) with amino-acid residues in the binding pocket of AR (right panel).

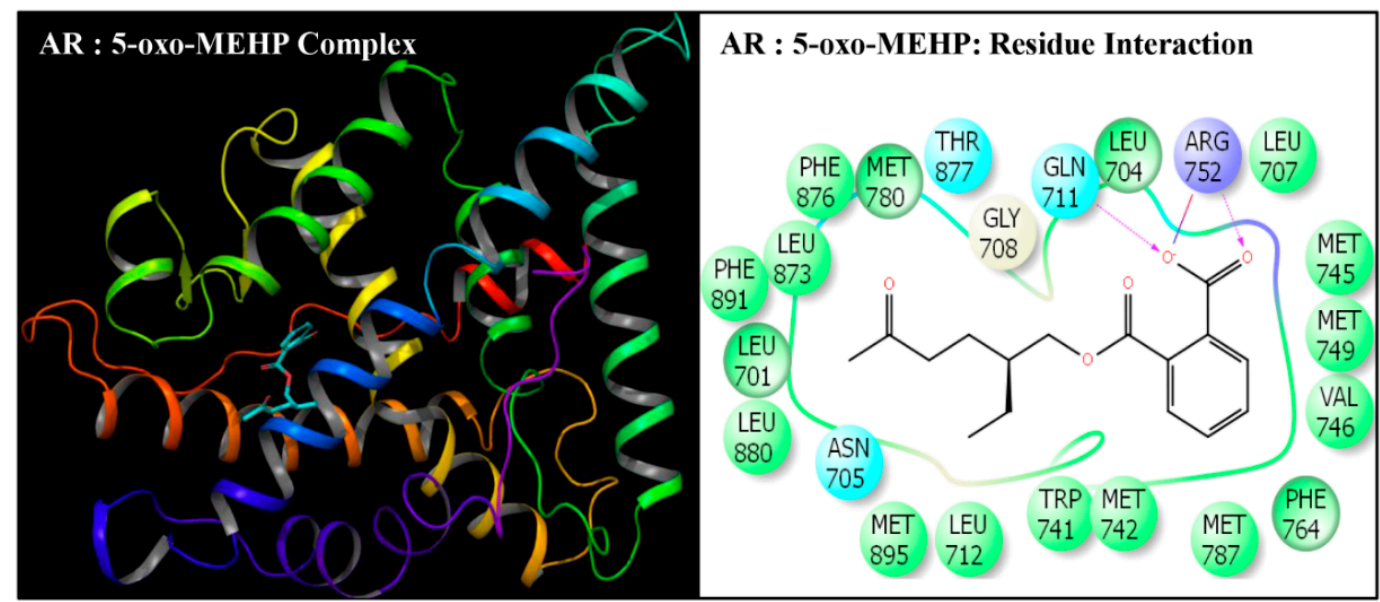

Figure 5. Ribbon diagram representing the docking pose of human androgen receptor (AR) with mono-(2-ethyl-5-oxohexyl)phthalate (5-oxo-MEHP) (left panel). The interactions of ligand (5-ox-MEHP) with amino-acid residues in the binding pocket of AR (right panel).

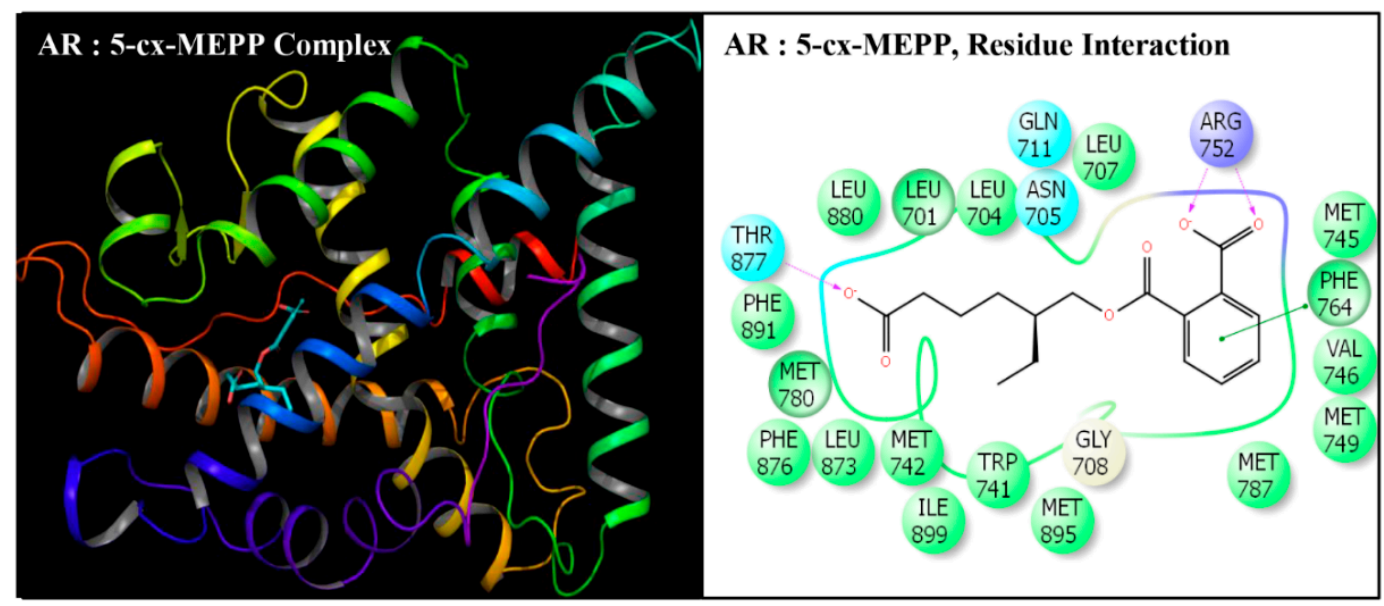

Figure 6. Ribbon diagram representing the docking pose of human androgen receptor (AR) with mono-(2-ethyl-5-carboxypentyl)phthalate (5-cx-MEPP) (left panel). The interactions of ligand (5-cx-MEPP) with amino-acid residues in the binding pocket of AR (right panel). 

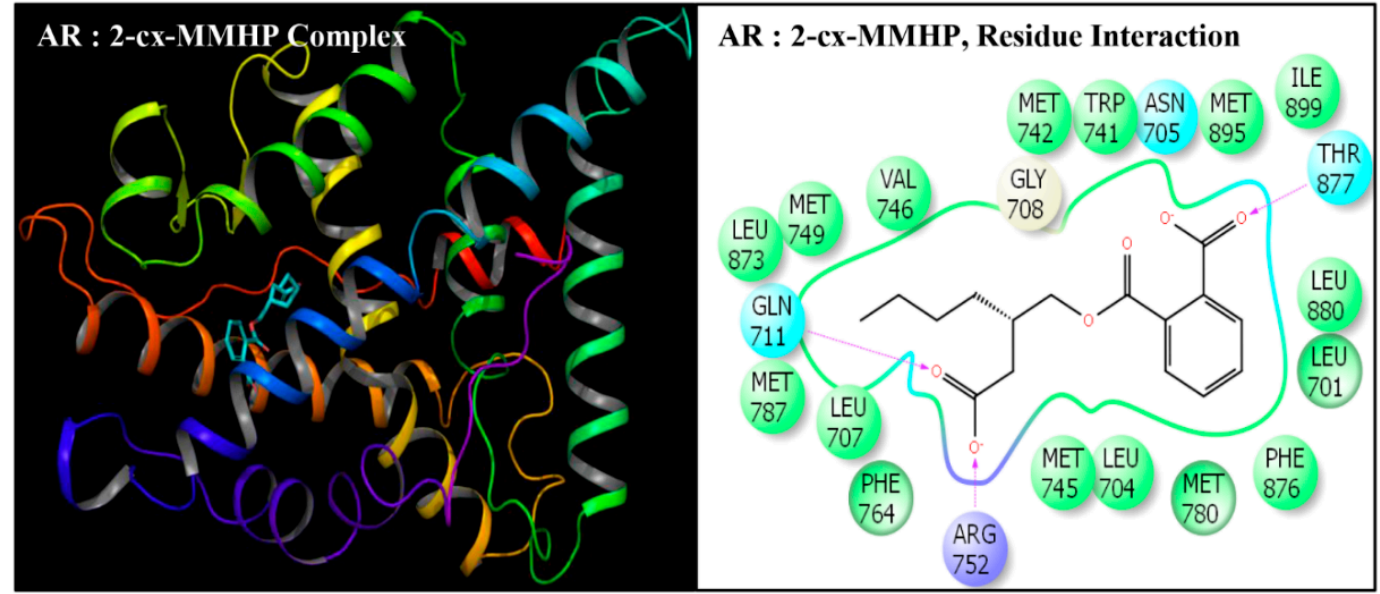

Figure 7. Ribbon diagram representing the docking pose of human androgen receptor (AR) with mono-[2-(carboxymethyl)hexyl]phthalate (2-cx-MMHP)(left panel). The interaction of ligand (2-cx-MMHP) with amino-acid residues in the binding pocket of AR (right panel).

Table 2. The number of interacting residues, number and percentage of residues common with native ligand testosterone (TST), Induced Fit Docking (IFD) Score, Dock score, Glide score, and binding affinity values (MMGB-SA values) of di(2-ethylhexyl)phthalate (DEHP), mono-(2-ethylhexyl)phthalate (MEHP), mono-(2-ethyl-5-hydroxyhexyl)phthalate (5-OH-MEHP), mono-(2-ethyl-5-oxohexyl)phthalate (5-oxo-MEHP), mono-(2-ethyl-5-carboxypentyl)phthalate (5-cx-MEPP), and mono-[2-(carboxymethyl)hexyl]phthalate (2-cx-MMHP) and native ligand, TST, after IDF with human androgen receptor (AR).

\begin{tabular}{cccccccc}
\hline Serial No. & Ligand & $\begin{array}{c}\text { Number of } \\
\text { Interacting AR } \\
\text { Residues }\end{array}$ & $\begin{array}{c}\text { Number of } \\
\text { Interacting } \\
\text { Residues } \\
\text { Common with } \\
\text { TST (\%) }\end{array}$ & IFD Score & $\begin{array}{c}\text { Docking } \\
\text { Score } \\
\text { (kcal/mol) }\end{array}$ & $\begin{array}{c}\text { Glide } \\
\text { Score } \\
\text { (kcal/mol) }\end{array}$ & $\begin{array}{c}\text { MMGB-SA } \\
\text { (kcal/mol) }\end{array}$ \\
\hline 1 & DEHP & 23 & $21(95 \%)$ & -573.86 & -9.17 & -9.17 & -136.12 \\
2 & MEHP & 21 & $21(95 \%)$ & -573.30 & -9.42 & -9.42 & -109.19 \\
3 & 5-OH-MEHP & 22 & $22(100 \%)$ & -573.33 & -9.65 & -9.65 & -107.50 \\
4 & 5-oxo-MEHP & 22 & $20(91 \%)$ & -574.45 & -9.73 & -9.73 & -105.80 \\
5 & 5-cx-MEPP & 21 & $22(100 \%)$ & -575.94 & -11.69 & -11.69 & -92.03 \\
6 & 2-cx-MMHP & 21 & $21(95 \%)$ & -574.48 & -10.41 & -10.41 & -90.60 \\
7 & TST & 22 & $22(100 \%)$ & -577.85 & -12.84 & -12.84 & -152.11 \\
\hline
\end{tabular}

Docking poses of six compounds (DEHP and five metabolites) in the ligand-binding domains of AR resulted in 21-23 amino-acid residue interactions (Figures 2-7, Table 2). Further, the docking complex of native ligand, TST, displayed interactions with 22 amino-acid residues of AR (Figure 8). The list of common interacting amino-acid residues for DEHP metabolites and TST is shown (Table 3). In addition, DEHP also interacted with Phe-770 and Val-889, while 5-oxo-MEHP also interacted with Leu-712. DEHP and its five metabolites shared all the amino acid residues that interacted with native ligand TST except that four amino-acid residues, i.e., Arg-752, Leu-880, Phe-891, and Ile-899 were not shared, respectively, by DEHP, MEHP, 2-cx-MMHP, and 5-oxo-MEHP (Table 3). Overall, there was a commonality of $91-100 \%$ of residues between DEHP compounds and the native ligand (Table 2). In addition to the amino acid interactions, DEHP metabolites also formed hydrogen bonds, pi-pi bond, and salt bridge interactions with AR. The metabolites MEHP, 5-OH-MEHP, 5-oxo-MEHP, and 2-cx-MMHP each formed two hydrogen bonds with amino-acid residues Gln-711 and Arg-752. Additionally, 5-OH-MEHP and 2-cx-MMHP each formed a hydrogen bond interaction with residue Thr-877. The metabolite 5-cx-MEPP did not form a hydrogen bond with Gln-711 but formed two hydrogen bonds with Arg-752 and one hydrogen bond with Thr-877. The native ligand TST also formed three hydrogen bonds with amino-acids Asn-705, Arg-752, and Thr-877. In addition to the 
hydrogen bond interactions, MEHP, 5-OH-MEHP, and 5-cx-MEPP each formed a pi-pi bond with Phe-764, and MEHP and 5-oxo-MEHP each formed a salt bridge interaction with Arg-752.

Table 3. Interacting amino-acid residues in the binding pocket of human androgen receptor that were common among natural ligand, testosterone, and di(2-ethylhexyl) phthalate (DEHP) and its five major metabolites, mono-(2-ethylhexyl)phthalate (MEHP), mono-(2-ethyl-5-hydroxyhexyl) phthalate (5-OH-MEHP), mono-(2-ethyl-5-oxohexyl)phthalate (5-oxo-MEHP), mono-(2-ethyl-5carboxypentyl)phthalate (5-cx-MEPP), and mono-[2-(carboxymethyl)hexyl]phthalate (2-cx-MMHP).

\begin{tabular}{cccc}
\hline Serial No. & Interacting Residue & Serial No. & Interacting Residue \\
\hline 1 & Leu-701 & 12 & Arg-752* \\
2 & Leu-704 & 13 & Phe-764 \\
3 & Asn-705 & 14 & Met-780 \\
4 & Leu-707 & 15 & Met-787 \\
5 & Gly-708 & 16 & Leu-873 \\
6 & Gln-711 & 17 & Phe-876 \\
7 & Trp-741 & 18 & Thr-877 \\
8 & Met-742 & 19 & Leu-880 \\
9 & Met-745 & 20 & Phe-891 \& \\
10 & Val-746 & 21 & Met-895 \\
11 & Met-749 & 22 & Ile-899 \# \\
\hline
\end{tabular}

Amino-acid residues indicated by superscripts were not shared DEHP $\left(^{*}\right), \operatorname{MEHP}\left({ }^{\$}\right), 2$-cx-MMHP $\left({ }^{\&}\right)$ and 5-oxo-MEHP $(\#)$.

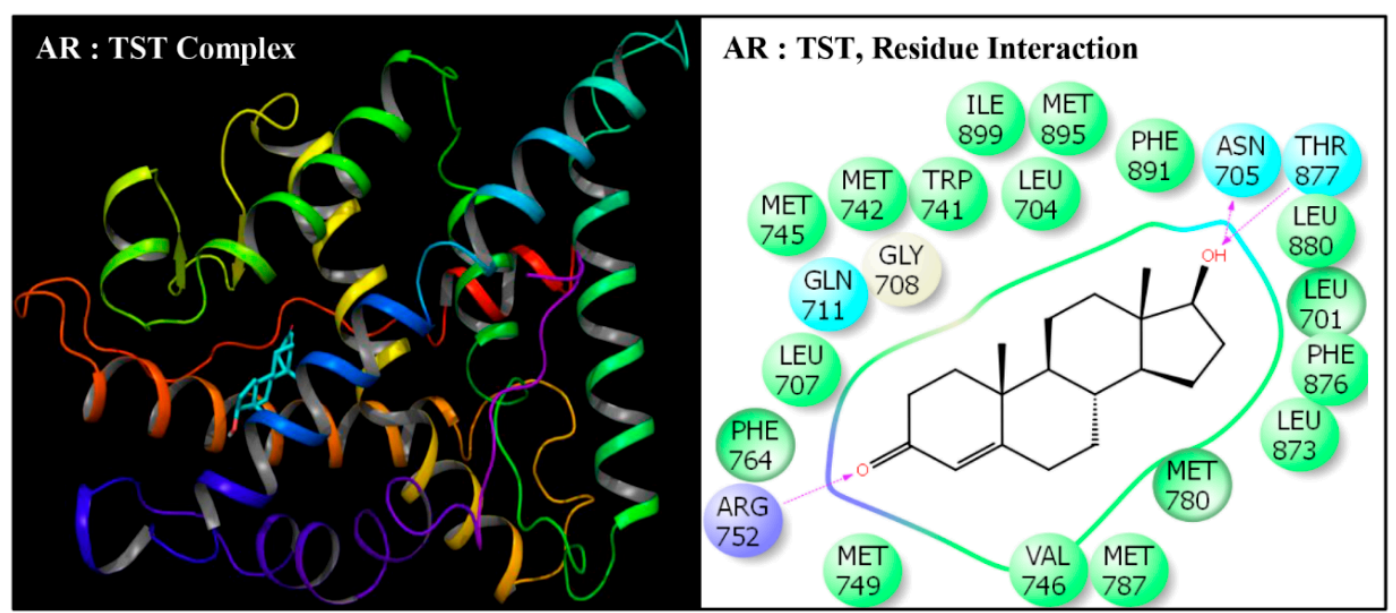

Figure 8. Ribbon diagram representing the docking pose of human androgen receptor (AR) with native ligand testosterone (TST) (left panel). The interactions of ligand (TST) with amino-acid residues in the binding pocket of AR (right panel).

The values for the Dock score, IFD score, Glide score, and binding affinity (MMGB-SA values) for all the ligands are shown (Table 2). The values for binding energy were highest for native ligand TST. The binding energy values for DEHP were close to that of TST, but the values for the five metabolites were lower (Table 2). The IFD scores were similar between five DEHP metabolites and native ligand TST. The Dock score and Glide score were highest for TST and lowest for DEHP, but progressively increased for MEHP, 5-OH-MEHP, 5-oxo-MEHP, and 5-cx-MEPP.

\section{Discussion}

The adult humans and newborns show a systemic predominance of the secondary metabolites of DEHP, which in comparison to the parent compound, remain in the system for a longer duration (20-22). In view of the toxic effects of parent compound DEHP, the present study was done to predict the potential adverse effects of the DEHP metabolites. The objective of the current research 
was to characterize the structure-based interactions of DEHP metabolites, i.e., MEHP, 5-OH-MEHP, 5-oxo-MEHP, 5-cx-MEPP, and 2-cx-MMHP with AR. The IFD approaches used in this study showed that DEHP and its metabolites docked deep in the ligand-binding domain of AR forming various interactions with a number of amino-acids. The molecular interactions between the ligands and the amino-acid residues in the AR ligand-binding pocket included hydrogen bonding, pi-pi, hydrophobic, salt bridge, etc. These interactions with the receptor anchored the ligands in the binding pocket. Analysis of the most stable docking pose for each ligand based on the result of cumulative analysis of docking parameters including amino acid interactions in the ligand-binding pocket of AR, and binding energy estimation revealed similar IFD score of DEHP ligands and native ligand TST together with good Glide and Dock score of the ligands indicating good strength and stability of the docking complexes. The closely similar values of binding energy between DEHP and TST also provided support for the good docking. For all the DEHP metabolites, the amino-acids interacting with AR were $95-100 \%$ common when compared with native ligand TST, which again indicated similarity in their structural and spatial conformation and, hence, manifested in docking. Further, a common hydrogen bonding interaction of DEHP metabolites and native ligand TST with amino-acid residue Arg-752 of AR also indicated a common binding mechanism of the DEHP metabolites and the native ligand. Additional hydrogen bonding interactions with amino-acid residues Gln-711 and Thr-877, pi-pi interactions with Phe-764, and salt bridge interaction with amino-acid residue Arg-752 of AR also provided support for the strength and stability of the binding of DEHP metabolites with AR. Hence, the similarity in docking and amino acid interactions between DEHP and its metabolites and native ligand TST with AR indicated the potential of DEHP metabolites for interference in the TST binding. A potential interference in binding may result in AR signaling disruption and abnormal androgen-related reproductive function.

The toxicological evaluation has been exclusively done for the parent compound DEHP, and very few studies have been conducted on DEHP metabolites, especially the secondary metabolites. Further, in vitro and in silico studies have been largely equivocal about the antagonistic activity of DEHP metabolites against AR [39-41]. In a recent study [34], using luciferase assays for analyzing the androgenic or antiandrogenic activity of DEHP and its important metabolites, it was shown that in contrast to the antiandrogenic activity of DEHP, the primary and secondary metabolites did not show any activity with AR. Whereas, in another recent study [35], using similar luciferase assays, neither DEHP nor its major primary and secondary metabolites showed any antagonistic activity against AR. This is in contrast to the structural binding results in the present study in which DEHP and its metabolites interacted with AR and were predicted to exert antagonistic effects. However, our results are supported by a study using yeast androgen bioassay (XenoScreen YES/YAS assay) in which metabolite MEHP was shown to exhibit anti-androgenic activity [36]. These inconsistent results were also observed in in silico docking studies. In one study [33], DEHP and its primary metabolite MEHP were reported to efficiently bind to human AR supporting our docking results in the present study. In addition, Leu-704, Asn-705, Gln-711, Arg-752, Phe-764, and Thr-877 were found as the crucial amino-acid residues in the ligand-binding pocket of AR in both the reported study and our study. In contrast, in another docking study, DEHP and its primary and secondary metabolites (MEHP, 5-OH-MEHP, 5-oxo-MEHP, 5-cx-MEPP, and 2-cx-MMHP) were reported to not interact with $A R$, and the study suggested a very low possibility of their antiandrogenic activity [35]. The differences in the results are not known. The direct in vivo toxicological experiments in laboratory animals on 5-OH-MEHP, 5-oxo-MEHP, 5-cx-MEPP, and 2-cx-MMHP are not available. In view of the previous equivocal results and lack of direct in vivo studies on the metabolites, further in vitro, in vivo, and structural studies on adverse effects of DEHP metabolites on androgen signaling are recommended to explore their endocrine-disrupting mechanisms. Special consideration must be given to DEHP metabolites in view of the ubiquitous nature of DEHP as even a weak significant association with adverse outcomes may be profoundly relevant for exposed populations. Computational models have very important relevance in the fast predictive assessment of the toxicity of environmental chemicals to 
human systems. It might be important to consider a combination of computational modelings such as pharmacophore screening, molecular docking, and MD simulations together with experimental assays for a holistic approach for more reliable prediction of AR disrupting risk of environmental compounds such as DEHP and its metabolites.

Epidemiological studies have associated DEHP as a cause for testicular dysgenesis syndrome manifested by infertility, genital developmental problems such as hypospadias, cryptorchidism, etc., and testicular cancer in exposed men $[42,43]$. Unborn and newborn babies being at maximum risk of adverse effects due to their immature metabolism and excretory systems. In this regard, some of the adverse effects of prenatal exposure are preterm birth, anogenital distance problems, low birth weight, low rate of weight gain, and effects on the femur, birth, head, biparietal, and abdominal dimensions in male and female babies [44]. DEHP exposure during gestation increased the risk of DNA methylation problems interfering in gene expression and adverse health conditions during childhood [45]. In addition, prenatal exposure was associated with aggressive behavior, defiance, and poor attention in children [46]. Precocious development such as early menarche of girls between 6-14 years of age and delayed menarche such as poor hair and testis development in boys have been associated with higher urinary MEHP levels [47]. In other studies, semen and urine levels of MEHP, 5-OH-MEHP, 5-oxo-MEHP, and DEHP metabolites excreted as percentage of MEHP predisposed to a low volume of semen, abnormal morphology, low motility, and lower testosterone levels in infertile men [48]. DEHP metabolites were also associated with high sperm DNA stainability indicating sperm immaturity [19]. In Japan, gestational circulatory MEHP levels were associated with lower concentrations of testosterone, progesterone, estradiol, cortisol, cortisone, insulin-like factor 3 and inhibin-B, and a lower ratio of glucocorticoid/adrenal androgen but higher concentrations of dehyroepiandrostenedione (DHEA) and a higher ratio of DHEA/androstenedione in cord blood of male newborns $[49,50]$. A recent study reported that in vitro exposure of human spermatozoa to DEHP and MEHP caused increased calcium in sperms and increased tyrosine phosphorylation. However, the significance of these effects is yet unknown [51].

In experimental studies on rats, pre and postnatal DEHP exposure have been associated with intrauterine mortality, impaired testicular development, teratogenicity, reduced anogenital distance, nipple retention, Leydig cell hyperplasia, hormone (especially testosterone) imbalance, and other reproductive system developmental problems even with relatively lower doses [52,53]. Particularly in male newborn rats, prenatal DEHP exposure causes congenital disabilities such as typical male dysgenesis or phthalate syndrome showing testis tubule atrophy, anogenital, epididymal, testicular, vas deferens, and external genitalia anomalies [54,55]. In adult male rats, DEHP is gonadotoxic and leads to testis atrophy, reduced litter size, lower sperm concentration, lower sperm motility, high sperm abnormalities, lower sperm fertilizing ability, zinc depletion, lower DNA replication, increased apoptosis, increase oxidative stress [56].

\section{Conclusions}

The current study prioritized the prediction of potential AR disrupting effects of DEHP metabolites by in silico structural interactions of the major endogenous hydroxylated and oxidative metabolites (MEHP, 5-OH-MEHP, 5-oxo-MEHP, 5-cx-MEPP, and 2-cx-MMHP) of DEHP with AR. The structural interaction data showed that DEHP and the five major metabolites have relatively strong binding affinities for AR. The binding energy values of DEHP were similar to that of native ligand TST. A very high (95-100\%) similarity was found in interacting AR amino-acid residues among DEHP metabolites and TST. In addition, hydrogen bonding between all the DEHP metabolites and amino-acid Arg-752 of AR was also common with native ligand TST. Taking into consideration the reported adverse effects of DEHP and similarity in docking and amino acid interactions among DEHP metabolites and the native ligand TST with AR, the data suggest a potential disrupting effect of DEHP metabolites on AR signaling, which may lead to abnormal androgen-related reproductive function. From public health perspectives, this study underscores the need for reduced use of plastic materials containing 
DEHP in industrial and commercial applications and spreading public awareness of their health hazards, especially in vulnerable pregnant women and newborn children. Further studies, especially computational modeling and in vitro and in vivo experiments, are recommended for predicting and assessing the AR-disrupting risk of environmental compounds such as DEHP and its metabolites.

Author Contributions: Conceptualization, M.A.B.; methodology, I.A.S.; software, I.A.S.; validation, I.A.S.; formal analysis, I.A.S.; data analysis, M.A.B. and I.A.S.; writing-original draft preparation, M.A.B.; writing-review and editing, I.A.S.; supervision, I.A.S.; project administration, M.A.B.; funding acquisition, M.A.B. Both authors have read and agreed to the published version of the manuscript.

Funding: Mohd A. Beg received funding for this study from Deanship of Scientific Research (DSR), King Abdulaziz University, Jeddah, (grant no. DF-331-141-1441).

Acknowledgments: This project was funded by the Deanship of Scientific Research (DSR), King Abdulaziz University, Jeddah, under grant no. (DF-331-141-1441). The authors, therefore, gratefully acknowledge DSR technical and financial support.

Conflicts of Interest: The authors declare no conflict of interest.

\section{References}

1. Dutta, S.; Haggerty, D.K.; Rappolee, D.A.; Ruden, D.M. Phthalate exposure and long-term epigenomic consequences: A review. Front. Genet. 2020, 11, 405. [CrossRef] [PubMed]

2. Environmental Protection Agency. Draft Scope of the Risk Evaluation for Di-ethylhexyl Phthalate (1,2-Benzenedicarboxylic acid, 1,2-bis(2-ethylhexyl) ester). The United States Environmental Protection Agency, Office of Chemical Safety and Pollution Prevention (OCSPP), Office of Pollution Prevention and Toxics (OPPT). EPA Document\# EPA-740-D-20-017. 2020; pp. 1-113. Available online: https://www.epa.gov/sites/production/ files/2020-04/documents/casrn_117-81-7-diethylhexyl_phthalate_draft_scope_4-15-2020.pdf (accessed on 7 December 2020).

3. Yuan, X.Q.; Du, Y.Y.; Liu, C.; Guo, N.; Teng, X.M.; Hua, X.; Yao, Y.C.; Deng, Y.L.; Zeng, Q.; Deng, T.R.; et al. Phthalate metabolites and biomarkers of oxidative stress in the follicular fluid of women undergoing in vitro fertilization. Sci. Total Environ. 2020, 738, 139834. [CrossRef] [PubMed]

4. Plastics Insight. Global Plasticizers Market. 2016. Available online: https://www.plasticsinsight.com/globalplasticisers-market/ (accessed on 7 December 2020).

5. Ceresana. Global demand for plasticizers continues to rise. Add. Polym. 2017, 10, 10-11.

6. Guo, Y.; Kannan, K. A survey of phthalates and parabens in personal care products from the United States and its implications for human exposure. Environ. Sci. Technol. 2013, 47, 14442-14449. [CrossRef]

7. Balalian, A.A.; Whyatt, R.M.; Liu, X.; Insel, B.J.; Rauh, V.A.; Herbstman, J.; Factor-Litvak, P. Prenatal and childhood exposure to phthalates and motor skills at age 11 years. Environ. Res. 2020,171, 416-427. [CrossRef]

8. Kahn, L.G.; Philippat, C.; Nakayama, S.F.; Slama, R.; Trasande, L. Endocrine-disrupting chemicals: Implications for human health. Lancet Diabetes Endocrinol. 2020, 8, 703-718. [CrossRef]

9. United States Environmental Protection Agency; Office of Chemical Safety and Pollution Prevention. Proposed Designation of di-ethylhexyl Phthalate (DEHP) (1,2-Benzene-dicarboxylic acid, 1,2-bis (2-ethylhexyl) Ester) (CASRN 117-81-7) as a High-Priority Substance for Risk Evaluation. 2019; pp. 1-59. Available online: https://www.epa.gov/sites/production/files/2019-08/documents/di-ethylhexyl_phthalate_117-81-7_ proposeddesignation_082219.pdf (accessed on 7 December 2020).

10. International Agency for Research on Cancer. Di(2-ethylhexyl)phthalate. In Some Chemicals Present in Industrial and Consumer Products, Food and Drinking-Water; IARC Monographs on the Evaluation of Carcinogenic Risks to Humans, No. 101. IARC Working Group on the Evaluation of Carcinogenic Risks to Humans; International Agency for Research on Cancer: Lyon, France, 2013; pp. 149-284. Available online: https://publications.iarc.fr/125 (accessed on 7 December 2020).

11. European Communities. Commission Directive 2001/59/EC of 6 August 2001 adapting to technical progress for the 28th time Council Directive 67/548/EEC on the approximation of the laws, regulations and administrative provisions relating to the classification, packaging and labelling of dangerous substances. Off. J. Eur. Communities 2001, L225, 1-333.

12. Japan Society for Occupational Health. Occupational exposure limit. Sangyo Eiseigaku Zasshi 2015, 57, 270-274. (In Japanese) 
13. Heudorf, U.; Mersch-Sundermann, V.; Angerer, J. Phthalates: Toxicology and exposure. Int. J. Hyg. Environ. Health 2007, 210, 623-634. [CrossRef]

14. Giuliani, A.; Zuccarini, M.; Cichelli, A.; Khan, H.; Reale, M. Critical review on the presence of phthalates in food and evidence of their biological impact. Int. J. Environ. Res. Public Health 2020, 17, 5655. [CrossRef]

15. Schecter, A.; Lorber, M.; Guo, Y.; Wu, Q.; Yun, S.; Kannan, K.; Hommel, M.; Imran, N.; Hynan, L.S.; Cheng, D.; et al. Phthalate concentrations and dietary exposure from food purchased in New York State. Environ. Health Perspect. 2013, 121, 473-479. [CrossRef] [PubMed]

16. Kaestner, F.; Seiler, F.; Rapp, D.; Eckert, E.; Müller, J.; Metz, C.; Bals, R.; Drexler, H.; Lepper, P.M.; Göen, T. Exposure of patients to di(2-ethylhexy)phthalate (DEHP) and its metabolite MEHP during extracorporeal membrane oxygenation (ECMO) therapy. PLOS ONE 2020, 15, e0224931. [CrossRef]

17. Apel, P.; Kortenkamp, A.; Koch, H.M.; Vogel, N.; Rüther, M.; Kasper-Sonnenberg, M.; Conrad, A.; Brüning, T.; Kolossa-Gehring, M. Time course of phthalate cumulative risks to male developmental health over a 27-year period: Biomonitoring samples of the German Environmental Specimen Bank. Environ. Int. 2020, 137, 105467. [CrossRef] [PubMed]

18. Koch, H.M.; Bolt, H.M.; Angerer, J. Di(2-ethylhexyl)phthalate (DEHP) metabolites in human urine and serum after a single oral dose of deuterium-labelled DEHP. Arch. Toxicol. 2004, 78, 123-130. [CrossRef] [PubMed]

19. Axelsson, J.; Rylander, L.; Rignell-Hydbom, A.; Lindh, C.H.; Jönsson, B.A.; Giwercman, A. Prenatal phthalate exposure and reproductive function in young men. Environ. Res. 2015, 138, 264-270. [CrossRef] [PubMed]

20. Koch, H.M.; Bolt, H.M.; Preuss, R.; Angerer, J. New metabolites of di(2-ethylhexyl)phthalate (DEHP) in human urine and serum after single oral doses of deuterium-labelled DEHP. Arch. Toxicol. 2005, 79, 367-376. [CrossRef] [PubMed]

21. Strømmen, K.; Lyche, J.L.; Blakstad, E.W.; Moltu, S.J.; Veierød, M.B.; Almaas, A.N.; Sakhi, A.K.; Thomsen, C.; Nakstad, B.; Brække, K.; et al. Increased levels of phthalates in very low birth weight infants with septicemia and bronchopulmonary dysplasia. Environ. Int. 2016, 89-90, 228-234. [CrossRef]

22. Stroustrup, A.; Bragg, J.B.; Busgang, S.A.; Andra, S.S.; Curtin, P.; Spear, E.A.; Just, A.C.; Arora, M.; Gennings, C. Sources of clinically significant neonatal intensive care unit phthalate exposure. J. Expo. Sci. Environ. Epidemiol. 2020, 30, 137-148. [CrossRef]

23. Lin, L.; Zheng, L.X.; Gu, Y.P.; Wang, J.Y.; Zhang, Y.H.; Song, W.M. Levels of environmental endocrine disruptors in umbilical cord blood and maternal blood of low-birth-weight infants. Zhonghua Yu Fang Yi Xue Za Zhi 2008, 42, 177-180.

24. Huang, P.C.; Kuo, P.L.; Chou, Y.Y.; Lin, S.J.; Lee, C.C. Association between prenatal exposure to phthalates and the health of newborns. Environ. Int. 2009, 35, 14-20. [CrossRef]

25. Krotz, S.P.; Carson, S.A.; Tomey, C.; Buster, J.E. Phthalates and bisphenol do not accumulate in human follicular fluid. J. Assist. Reprod. Genet. 2012, 29, 773-777. [CrossRef] [PubMed]

26. Hogberg, J.; Hanberg, A.; Berglund, M.; Skerfving, S.; Remberger, M.; Calafat, A.M.; Filipsson, A.F.; Jansson, B.; Johansson, N.; Appelgren, M.; et al. Phthalate diesters and their metabolites in human breast milk, blood or serum, and urine as biomarkers of exposure in vulnerable populations. Environ. Health Perspect. 2008, 116, 334-339. [CrossRef] [PubMed]

27. Kavlock, R.; Boeckelheide, K.; Chapin, R.; Cunningham, M.; Faustman, E.; Foster, P.; Golub, M.; Henderson, R.; Hinberg, I.; Little, R.; et al. NTP Center for the Evaluation of Risks to Human Reproduction: Phthalates expert panel report on the reproductive and developmental toxicity of di(2-ethylhexyl)phthalate. Reprod. Toxicol. 2002, 16, 529-653. [CrossRef]

28. Regnier, J.; Bowden, C.; Lhuguenot, J. Effects on rat embryonic development in vitro of di-(2-ethylhexyl) phthalate (DEHP) and its metabolites. Toxicologist 2004, 78, 38-39.

29. Swan, S.H.; Sathyanarayana, S.; Barrett, E.S.; Janssen, S.; Liu, F.; Nguyen, R.H.; Redmon, J.B.; TIDES Study Team. First trimester phthalate exposure and anogenital distance in newborns. Hum. Reprod. 2015, 30, 963-972. [CrossRef]

30. Huang, L.P.; Lee, C.C.; Fan, J.P.; Kuo, P.H.; Shih, T.S.; Hsu, P.C. Urinary metabolites of di(2-ethylhexyl) phthalate relation to sperm motility, reactive oxygen species generation, and apoptosis in polyvinyl chloride workers. Int. Arch. Occup. Environ. Health 2014, 87, 635-646. [CrossRef]

31. Demirel, A.; Çoban, A.; Yıldırım, S.; Doğan, C.; Sancı, R.; İnce, Z. Hidden toxicity in neonatal intensive care units: Phthalate exposure in very low birth weight infants. J. Clin. Res. Pediatr. Endocrinol. 2016, 8, 298-304. [CrossRef] 
32. Gore, A.C.; Crews, D.; Doan, L.L.; La Merrill, M.; Patisaul, H.; Zota, A. Introduction to Endocrine Disrupting Chemicals (EDCs) - A Guide for Public Interest Organizations and Policy Makers. Endocrine Society Reports and White Papers. 2014, pp. 1-76. Available online: https://www.endocrine.org/-/media/endosociety/ files/advocacy-and-outreach/important-documents/introduction-to-endocrine-disrupting-chemicals.pdf (accessed on 7 December 2020).

33. Sarath Josh, M.K.; Pradeep, S.; Vijayalekshmy Amma, K.S.; Sudha Devi, R.; Balachandran, S.; Sreejith, M.N.; Benjamin, S. Human ketosteroid receptors interact with hazardous phthalate plasticizers and their metabolites: An in silico study. J. Appl. Toxicol. 2016, 36, 836-843. [CrossRef]

34. Engel, A.; Buhrke, T.; Imber, F.; Jessel, S.; Seidel, A.; Völkel, W.; Lampen, A. Agonistic and antagonistic effects of phthalates and their urinary metabolites on the steroid hormone receptors ERalpha, ERbeta, and AR. Toxicol. Lett. 2017, 277, 54-63. [CrossRef]

35. Kambia, N.K.; Séverin, I.; Farce, A.; Moreau, E.; Dahbi, L.; Duval, C.; Dine, T.; Sautou, V.; Chagnon, M.C. In vitro and in silico hormonal activity studies of di-(2-ethylhexyl)terephthalate, a di-(2-ethylhexyl)phthalate substitute used in medical devices, and its metabolites. J. Appl. Toxicol. 2019, 39, 1043-1056. [CrossRef]

36. Kim, D.H.; Park, C.G.; Kim, S.H.; Kim, Y.J. The effects of mono-(2-ethylhexyl) phthalate (MEHP) on human estrogen receptor (hER) and androgen receptor (hAR) by YES/YAS in vitro assay. Molecules 2019, 24, 1558. [CrossRef] [PubMed]

37. Sheikh, I.A.; Turki, R.F.; Abuzenadah, A.M.; Damanhouri, G.A.; Beg, M.A. Endocrine disruption: Computational perspectives on human sex hormone-binding globulin and phthalate plasticizers. PLoS ONE 2016, 11, e0151444. [CrossRef] [PubMed]

38. Nabuurs, S.B.; Wagener, M.; de Vlieg, J. A flexible approach to induced fit docking. J. Med. Chem. 2007, 50, 6507-6518. [CrossRef] [PubMed]

39. Araki, N.; Ohno, K.; Nakai, M.; Takeyoshi, M.; Lida, M. Screening for androgen receptor activities in 253 industrial chemicals by in vitro reporter gene assays using AR-EcoScreen cells. Toxicol. In Vitro 2005, 19, 831-842. [CrossRef] [PubMed]

40. Krüger, T.; Long, M.; Bonefeld-Jørgensen, E.C. Plastic components affect the activation of the aryl hydrocarbon and the androgen receptor. Toxicology 2008, 246, 112-123. [CrossRef] [PubMed]

41. Shen, O.; Du, G.; Sun, H.; Wu, W.; Jiang, Y.; Song, L.; Wang, X. Comparison of in vitro hormone activities of selected phthalates using reporter gene assays. Toxicol. Lett. 2009, 191, 9-14. [CrossRef] [PubMed]

42. Jensen, M.S.; Anand-Ivell, R.; Nørgaard-Pedersen, B.; Jönsson, B.A.G.; Bonde, J.P.; Hougaard, D.M.; Cohen, A.; Lindh, C.H.; Ivell, R.; Toft, G. Amniotic fluid phthalate levels and male fetal gonad function. Epidemiology 2015, 26, 91-99. [CrossRef]

43. Jurewicz, J.; Radwan, M.; Sobala, W.; Ligocka, D.; Radwan, P.; Bochenek, M.; Jakubowski, L.; Hanke, W. Human urinary phthalate metabolites level and main semen parameters, sperm chromatin structure, sperm aneuploidy and reproductive hormones. Reprod. Toxicol. 2013, 42, 232-241. [CrossRef]

44. Katsikantami, I.; Sifakis, S.; Tzatzarakis, M.N.; Vakonaki, E.; Kalantzi, O.I.; Tsatsakis, A.M.; Rizos, A.K. A global assessment of phthalates burden and related links to health effects. Environ. Int. 2016, 97, 12-236. [CrossRef]

45. Huen, K.; Calafat, A.M.; Bradman, A.; Yousefi, P.; Eskenazi, B.; Holland, N. Maternal phthalate exposure during pregnancy is associated with DNA methylation of LINE-1 and Alu repetitive elements in Mexican-American children. Environ. Res. 2016, 148, 55-62. [CrossRef]

46. Lien, Y.J.; Ku, H.Y.; Su, P.H.; Chen, S.J.; Chen, H.Y.; Liao, P.C.; Chen, W.J.; Wang, S.L. Prenatal exposure to phthalate esters and behavioral syndromes in children at 8 years of age: Taiwan maternal and infant cohort study. Environ. Health Perspect. 2015, 123, 95-100. [CrossRef] [PubMed]

47. Shi, H.; Cao, Y.; Shen, Q.; Zhao, Y.; Zhang, Z.; Zhang, Y. Association between urinary phthalates and pubertal timing in Chinese adolescents. J. Epidemiol. 2015, 25, 574-582. [CrossRef] [PubMed]

48. Wang, Y.; Zhu, H.; Kannan, K. A review of biomonitoring of phthalate exposures. Toxics 2019, 7, 21. [CrossRef]

49. Araki, A.; Mitsui, T.; Goudarzi, H.; Nakajima, T.; Miyashita, C.; Itoh, S.; Sasaki, S.; Cho, K.; Moriya, K.; Sinohara, N.; et al. Prenatal di(2-ethylhexyl) phthalate exposure and disruption of adrenal androgens and glucocorticoids levels in cord blood: The Hokkaido Study. Sci. Total Environ. 2017, 581-582, 297-304. [CrossRef]

50. Araki, A.; Mitsui, T.; Miyashita, C.; Nakajima, T.; Naito, H.; Ito, S.; Sasaki, S.; Cho, K.; Ikeno, T.; Nonomura, K.; et al. Association between maternal exposure to di(2-ethylhexyl) phthalate and reproductive 
hormone levels in fetal blood: The Hokkaido Study on Environment and Children's Health. PLoS ONE 2014, 9, e109039. [CrossRef] [PubMed]

51. Sun, X.; Chen, W.; Weng, S.; Pan, T.; Hu, X.; Wang, F.; Xia, T.; Chen, H.; Luo, T. Effects of the environmental endocrine disruptors di-2-ethylhexyl phthalate and mono-2-ethylhexyl phthalate on human sperm function invitro. Reprod. Fertil. Dev. 2020, 32, 629-636. [CrossRef] [PubMed]

52. Saillenfait, A.M.; Roudot, A.C.; Gallissot, F.; Sabate, J.P. Prenatal developmental toxicity studies on di-n-heptyl and di-n-octyl phthalates in Sprague-Dawley rats. Reprod. Toxicol. 2011, 32, 268-276. [CrossRef]

53. Zarean, M.; Keikha, M.; Poursafa, P.; Khalighinejad, P.; Amin, M.; Kelishadi, R. A systematic review on the adverse health effects of di-2-ethylhexyl phthalate. Environ. Sci. Pollut. Res. Int. 2016, 23, 24642-24693. [CrossRef]

54. Kay, V.R.; Bloom, M.S.; Foster, W.G. Reproductive and developmental effects of phthalate diesters in males. Crit. Rev. Toxicol. 2014, 44, 467-498. [CrossRef]

55. Lioy, P.J.; Hauser, R.; Gennings, C.; Koch, H.M.; Mirkes, P.E.; Schwetz, B.A.; Kortenkamp, A. Assessment of phthalates/phthalate alternatives in children's toys and childcare articles: Review of the report including conclusions and recommendation of the Chronic Hazard Advisory Panel of the Consumer Product Safety Commission. J. Expo. Sci. Environ. Epidemiol. 2015, 25, 343-353. [CrossRef]

56. Rowdhwal, S.S.S.; Chen, J. Toxic Effects of Di-2-ethylhexyl Phthalate: An Overview. BioMed Res. Int. 2018, 2018, 1750368. [CrossRef] [PubMed]

Publisher's Note: MDPI stays neutral with regard to jurisdictional claims in published maps and institutional affiliations. 